\title{
The development of a patient and caregiver narrative archive to support patient-centered research
}

\author{
Kathleen M McTigue MD MPH ${ }^{a}$, David Fear BS ${ }^{b}$, Ken Hunter BS ${ }^{c}$, Srimukhi Karanam BS , \\ James Uhrig BS ${ }^{e}$, Treva Alston $\mathrm{MS}^{f}$, Stacey Dillon MS MA ${ }^{g}$, Jenni Faust IB ${ }^{\mathrm{h}}$, Juliana Kim BS ${ }^{i}$, \\ Anthony LaRosa BS ${ }^{j}$, Barbara Postol PhD ${ }^{\mathrm{k}}$, Sylvia Umegbolu BS and Megan Hamm PhD ${ }^{\mathrm{m}}$
}

\author{
a Associate Professor of Medicine, Division of General Internal Medicine, University of Pittsburgh, Pittsburgh, PA, USA \\ b Medical Student, Geisinger Commonwealth School of Medicine, Scranton, PA, USA \\ c Research Assistant, Division of General Internal Medicine, University of Pittsburgh, Pittsburgh, PA, USA \\ d Medical Student, Ross University School of Medicine, Portsmouth, Dominica \\ e Patient Partner, PaTH Network, University of Pittsburgh, McMurray, PA, USA \\ f Patient Partner, PaTH Network, Johns Hopkins University, Laurel, MD, USA \\ g Behavioral Therapist, Division of General Internal Medicine, Pittsburgh, PA, USA \\ h Research Assistant, Division of General Internal Medicine, University of Pittsburgh, Pittsburgh, PA, USA \\ i Research Assistant, School of Dental Medicine-Dental Hygiene, University of Pittsburgh, Pittsburgh, PA, USA \\ j Mental Health Technician, Division of General Internal Medicine, University of Pittsburgh, Pittsburgh, PA, USA \\ k Project Manager, Division of General Internal Medicine, University of Pittsburgh, Pittsburgh, PA, USA \\ I Research Assistant, Division of General Internal Medicine, University of Pittsburgh, Pittsburgh, PA, USA \\ $\mathrm{m}$ Assistant Professor of Medicine \& Director of Qualitative, Evaluation and Stakeholder Engagement Research Services \\ (QualEASE), Division of General Internal Medicine, University of Pittsburgh, Pittsburgh, PA, USA
}

\begin{abstract}
Background: Engaging patients and other stakeholders is a key tenet of patient-centered research, but can be challenging to implement.

Objectives: To determine the feasibility of using patient narratives for informing comparative effectiveness research and engaging patients in the research process.

Research Design: Cross-sectional study.

Participants: The first 153 participants to share a story using the MyPaTH Story Booth protocol were, on average, aged 51 (18.8). Most were white (79\%) or African American (13\%) and well-educated.

Measures: Participants self-categorized their narratives according to the sort of health experience(s) described (e.g., diagnosis, doctor/patient communication), the anatomic locations of health problems discussed and the type of disorder or condition addressed. They also reported on whether they were interested in engaging in clinical research as a participant or stakeholder and their satisfaction with the study processes.

Results: The archived narratives addressed a wide range of health experiences of relevance to clinical researchers, most often medical treatment (82\%), communication with healthcare team members (81\%), diagnosis (64\%), symptoms (61\%), healthcare access (60\%) and health decision-making (60\%). Health problems related to diverse anatomic locations were addressed. Health conditions such as mental health concerns (35\%), infections (23\%) and cancers (20\%) were often discussed. Among participants, 63\% expressed interest in learning of opportunities for stakeholder engagement in research related to their stories. Ninety seven percent were very satisfied or satisfied with the MyPaTH Story Booth process.

Conclusions: The narrative archive represents a unique resource for researchers interested in developing and carrying out patient-centered research projects to improve health or healthcare.
\end{abstract}

\section{Keywords}

Narratives, patient-centered outcomes research, person-centered healthcare, stakeholder engagement

\section{Correspondence address}

Dr. Kathleen M. McTigue, 230 McKee Place, Suite 600, Division of General Internal Medicine, University of Pittsburgh, Pittsburgh, PA 15213, USA. E-mail: kmm34@pitt.edu

Accepted for publication: 20 March 2018 


\section{Introduction}

Engaging patients and other healthcare stakeholders in all aspects of the research process is a key tenet of patientcentered research [1,2]. Stakeholder input can inform the development of relevant research questions, methods and outcomes [1,3]. Early involvement of stakeholders holds particular promise for fostering research that addresses topics of relevance to patients and healthcare systems [4] and so may lead to improvements in healthcare delivery. Yet, at the time research is planned, it remains challenging for researchers to engage patients, as funding mechanisms and the culture of academic medicine often lack support for engagement activities [3,5]. The challenge is even greater for researchers who work in non-clinical settings, since they may not have routine exposure to patient or caregiver perspectives. Furthermore, patients or caregivers who lack technical training in patient-centered outcomes research (PCOR) may find it difficult to formulate technical research questions and communicate with researchers.

The goal of using patient stories to support patientcentered research is consistent with the literature which shows that patient stories can contribute to patient-centered care [6]. Narratives can convey complex technical and contextual knowledge to inform better health decisionmaking and help patients make sense of illness, while eliciting empathy and compassion [7-10]. Patient stories have supported self-management efforts [11,12] and health-related behavior change [13]. Narratives have likewise informed projects to improve the quality of care $[14,15]$, educate medical students about what it is like to live with illness [16] and share successful coping strategies for dealing with a health problem [17].

Insight into the experience of illness or seeking healthcare could be invaluable for identifying quality gaps in healthcare delivery or planning for improving processes of care. For example, one study found that patient stories can be categorized as factual (e.g., describing symptoms or the process of diagnosis or treatment), emotional (individuals' reactions to their health experiences) or moral (how people want to be perceived or feel they ought to be perceived) [10]. Another study that used narratives to understand the experience of cancer care found that patient stories addressed accountability (maintaining personal accountability or wanting others to take responsibility on their behalf), personal identity and life context (e.g., making sense of all that had happened) [14]. In addition, patient stories typically employed a non-linear construct of time and highlighted the importance of language - either in terms of a need for simple, non-technical communication, or mastery of technical language as a source of control. Logic and emotion may co-exist in patient stories, more so than in stories told by clinicians [14].

To gain insight into the feasibility of using narratives to support PCOR, the PaTH Clinical Data Research Network (CDRN) [18], a member of the United States' National Patient-Centered Clinical Research Network (PCORnet), established an audio-archive of patient and caregiver stories. The project aims to help inform the development of patient-centered research questions and facilitate the engagement of patients into the research process. Here, we report on the sample description, narrative categorization, interest in engaging in research and satisfaction data for the first 153 participants.

\section{Methods}

Adults aged 18 or above were invited to record a personal narrative about their experiences with health or illness. We used printed and internet-based advertisements to recruit from clinical and community venues and research registries. Participants were required to be able to read and understand English. Stories could address preventive health, dealing with illness, or experiences with healthcare or healthcare access. Patient or caregiver perspectives were welcomed.

Each narrative was elicited as a conversation with a friend, family member, or a member of the study's staff (based on participant preference). Consent was obtained electronically and data collection was completed over the telephone or in-person at a sound booth in the lobby of a medical building. This location was selected to be convenient for patients seeking routine medical care and accessible for those with mobility limitations. Interested individuals could schedule recording appointments ahead of time, or use “drop-in” hours to record a story without advance scheduling. Prior to story collection, a brief questionnaire assessed participants' demographics and determined whether participants were interested in hearing of research opportunities related to their story, either as a research participant or an engaged stakeholder. Respondents also reported preferences about potentially having their story posted online.

Participants were asked to limit their storytelling to 20 minutes and to avoid mentioning any identifying information. They were provided with a list of optional conversation prompts (i.e., potential interview questions) that they could use to direct the discussion towards topics of interest. Following the interview, participants were asked whether they approved the recording. If not, they were able to re-record. After approving a story, participants completed survey items to categorize the story's content in terms of the type of health experiences it described (e.g., finding out a diagnosis, communicating with health professionals, providing support for family members). In addition, the U.S. National Library of Medicine's categorization of health information topics for patients and their families (i.e., "body locations/systems" and "disorders and conditions") [19] were adapted for patients to identify the anatomic location of any health problems described in the story and whether the story addressed a health problem of relevance to multiple organ systems (e.g., cancer, infectious disease) or an issue that, while not a health problem, does impact health (e.g., pregnancy).

If participants reported that their story was related to a topic addressed by a partnering PCORNet Patient Powered Research Network (PPRN), they were given options for 
learning more about that organization. Further survey items noted whether the story addressed their own health or their role as a caregiver, addressed satisfaction with the narrative recording process and provided an opportunity to suggest process improvements.

Research staff reviewed each story, using standard protocols to identify and obscure potentially identifying information (e.g., personal names, location, facility names, proprietary drug or device names). They also completed the same questions that participants had answered to categorize the story's content and viewpoint.

The PaTH Clinical Data Research Network patient partners provided input on whether to propose the narrative collection project and how to implement it. The project was approved by The PaTH Clinical Data Research Network Protocol Review Committee and the University of Pittsburgh Institutional Review Board (PRO15100466).

\section{Results}

Participants were, on average, 51 years of age and 65\% were female (Table 1). Most (84\%) had attended or graduated from college. The sample was predominantly white (79\%) or African American (13\%).

Table 1 Sample description $(n=153)$

\begin{tabular}{|c|c|c|c|}
\hline & & $\begin{array}{c}\text { Mean } \\
\text { SD }\end{array}$ & $\begin{array}{c}\text { Frequency } \\
(\%)\end{array}$ \\
\hline Age & & 51 & $(18.8)$ \\
\hline \multicolumn{4}{|l|}{ Sex } \\
\hline & Male & 53 & (34.6) \\
\hline & Female & 100 & $(65.4)$ \\
\hline \multicolumn{4}{|l|}{ Education } \\
\hline & $8^{\text {th }}$ grade or less & 0 & $(0)$ \\
\hline & $\begin{array}{l}\text { Some high school but did } \\
\text { not graduate }\end{array}$ & 3 & (2) \\
\hline & $\begin{array}{l}\text { High school graduate or } \\
\text { GED }\end{array}$ & 21 & (13.7) \\
\hline & $\begin{array}{l}\text { Some college or 2-year } \\
\text { degree }\end{array}$ & 54 & (35.3) \\
\hline & College graduate & 34 & $(22.2)$ \\
\hline & $\begin{array}{l}\text { More than a college } \\
\text { degree }\end{array}$ & 40 & $(26.1)$ \\
\hline & Prefer not to answer & 1 & $(0.7)$ \\
\hline \multicolumn{4}{|l|}{$\begin{array}{l}\text { Race and } \\
\text { Ethnicity* }\end{array}$} \\
\hline & Asian & 3 & (2) \\
\hline & $\begin{array}{l}\text { Black, African American, } \\
\text { African or Afro- } \\
\text { Caribbean }\end{array}$ & 20 & $(13.1)$ \\
\hline & $\begin{array}{l}\text { Hispanic, Latino or } \\
\text { Spanish origin }\end{array}$ & 8 & (5.2) \\
\hline & $\begin{array}{l}\text { Middle Eastern/North } \\
\text { African }\end{array}$ & 1 & $(0.7)$ \\
\hline & $\begin{array}{l}\text { Native American, } \\
\text { American Indian or } \\
\text { Alaskan Native }\end{array}$ & 3 & (2) \\
\hline & $\begin{array}{l}\text { Native Hawaiian or Other } \\
\text { Pacific Islander }\end{array}$ & 0 & $(0)$ \\
\hline & White & 121 & (79.1) \\
\hline & $\begin{array}{l}\text { Some other race or origin } \\
\text { (please specify) }\end{array}$ & 1 & $(0.7)$ \\
\hline & Prefer not to answer & 1 & $(0.7)$ \\
\hline \multicolumn{4}{|l|}{ Perspective } \\
\hline & Patient & 125 & (81.7) \\
\hline & Caregiver & 28 & (18.3) \\
\hline
\end{tabular}

*Participants could identify all relevant options
Table 2 Summary of recorded narratives' content, as identified by the story tellers

\begin{tabular}{|c|c|c|}
\hline \multirow{2}{*}{$\begin{array}{l}\text { Type of healthcare experience(s) } \\
\text { described* } \\
\text { Being treated }\end{array}$} & \multicolumn{2}{|c|}{ Frequency (\%) } \\
\hline & 125 & $(81.7)$ \\
\hline $\begin{array}{l}\text { Communicating with doctors and } \\
\text { nurses }\end{array}$ & 124 & $(81.1)$ \\
\hline Finding out a diagnosis & 98 & $(64.1)$ \\
\hline Experiencing symptoms & 93 & $(60.8)$ \\
\hline $\begin{array}{l}\text { Describing how easy or hard it has } \\
\text { been for you to get the healthcare you } \\
\text { need }\end{array}$ & 91 & $(59.5)$ \\
\hline $\begin{array}{l}\text { Making decisions about the right } \\
\text { healthcare or treatment for you }\end{array}$ & 91 & $(59.5)$ \\
\hline $\begin{array}{l}\text { Getting tests or advice for how to be } \\
\text { healthier }\end{array}$ & 72 & $(47.1)$ \\
\hline $\begin{array}{l}\text { Supporting family members who are } \\
\text { sick }\end{array}$ & 50 & $(32.7)$ \\
\hline $\begin{array}{l}\text { Getting healthcare that is right for your } \\
\text { cultural or religious beliefs }\end{array}$ & 14 & $(9.2)$ \\
\hline Other & 14 & $(9.2)$ \\
\hline \multicolumn{3}{|l|}{$\begin{array}{l}\text { Anatomic location of health problems in } \\
\text { the narrative* }\end{array}$} \\
\hline Bones, joints and muscles & 53 & $(34.6)$ \\
\hline Brain and nerves & 53 & $(34.6)$ \\
\hline Blood, heart and circulation & 36 & $(23.5)$ \\
\hline Immune system & 35 & $(22.9)$ \\
\hline Lungs and breathing & 31 & $(20.3)$ \\
\hline Endocrine System & 26 & (17) \\
\hline Female reproductive system & 22 & $(14.4)$ \\
\hline Digestive system & 19 & $(12.4)$ \\
\hline Kidneys and urinary system & 17 & $(11.1)$ \\
\hline Eyes and vision & 15 & $(9.8)$ \\
\hline Skin, hair and nails & 12 & (7.8) \\
\hline Ear, nose and throat & 8 & $(5.2)$ \\
\hline Mouth and teeth & 7 & (4.6) \\
\hline Male reproductive system & 6 & (3.9) \\
\hline Other & 26 & $(17)$ \\
\hline \multicolumn{3}{|l|}{ Broader health issues addressed* } \\
\hline Mental Health & 54 & $(35.3)$ \\
\hline Infections & 35 & $(22.9)$ \\
\hline Cancers & 31 & $(20.3)$ \\
\hline Diabetes & 22 & $(14.4)$ \\
\hline Obesity & 18 & $(11.8)$ \\
\hline Substance Abuse & 13 & $(8.5)$ \\
\hline Genetics/Birth Defects & 13 & $(8.5)$ \\
\hline Poisoning or toxins in the environment & 8 & $(5.2)$ \\
\hline Pregnancy and Reproduction & 11 & $(7.2)$ \\
\hline Injuries and Wounds & 0 & (0) \\
\hline None of the above & 28 & $(18.3)$ \\
\hline
\end{tabular}

*Participants could identify all relevant options

Self-identified story topics included a wide range of issues related to healthcare delivery (Table 2). Stories most often included a focus on treatment (82\%), communication with healthcare team members (81\%), diagnosis (64\%), symptoms (61\%), healthcare access (60\%) and health decision-making (60\%). Narratives addressed health problems related to diverse anatomic locations, particularly musculoskeletal (35\%), cardiovascular or hematologic (35\%), neurologic (35\%), immunologic (23\%) and pulmonary (20\%) topics. Cross-cutting topics, not linked with a specific anatomic location, were quite common, 
such as mental health concerns (35\%), infections (23\%) and cancer (20\%).

Most participants provided permission for posting their story on the network's website (85\%). Many participants were interested in becoming engaged in clinical research. For example, $69 \%$ reported that they would like to hear about opportunities to work with researchers on a research project related to their stories. Likewise, 63\% expressed interest in specifically learning about opportunities to partner as a stakeholder in research related to their story.

Overall, $97 \%$ of participants were very satisfied or satisfied with the story collection process (Table 3 ). The experience of recording a narrative was described as easy or very easy by $86 \%$, while $9 \%$ were neutral and $5 \%$ reported that it was difficult. Eighty four percent of participants were very likely or likely to tell a friend about the MyPaTH Story Book initiative.

\section{Table 3 Participants' perception of the narrative collection process}

\begin{tabular}{|lcc|}
\hline $\begin{array}{l}\text { Level of satisfaction with the } \\
\text { MyPaTH Story Booth process }\end{array}$ & \multicolumn{2}{c|}{ Frequency } \\
$\quad$ Very satisfied & 121 & $(79.1)$ \\
Satisfied & 27 & $(17.7)$ \\
Neutral & 4 & $(2.6)$ \\
$\quad$ Slightly satisfied & 1 & $(0.7)$ \\
$\quad$ Not at all satisfied & 0 & $(0)$ \\
\hline $\begin{array}{l}\text { Description of the experience } \\
\text { of recording a narrative }\end{array}$ & & \\
$\quad$ Very easy & 96 & $(62.8)$ \\
$\quad$ Easy & 35 & $(22.9)$ \\
$\quad$ Neutral & 14 & $(9.2)$ \\
$\quad$ Difficult & 8 & $(5.2)$ \\
$\quad$ Very difficult & 0 & $(0)$ \\
\hline $\begin{array}{l}\text { Likelihood of telling a friend } \\
\text { about the MyPaTH Story }\end{array}$ & & \\
Booth story project & & \\
$\quad$ Very likely & 89 & $(58.2)$ \\
Likely & 40 & $(26.1)$ \\
Neutral & 18 & $(11.8)$ \\
Somewhat unlikely & 3 & $(2)$ \\
Very unlikely & 3 & $(2)$ \\
\hline
\end{tabular}

\section{Disc ussion}

The MyPaTH Story Booth archive of patient narratives includes stories describing a wide range of healthcare experiences as well as diverse health problems. This range suggests that the audio archive represents a unique resource for researchers interested in formulating patientcentered research questions or carrying out stakeholderengaged research that can lead to improvements in health or healthcare.

Our data indicate that a narrative archive can foster patient interest in engaging with PCOR and provide a mechanism for forming partnerships between researchers and patients or caregivers. For example, over half of the MyPaTH Story Booth participants expressed an interest in learning about research or stakeholder opportunities related to their stories. Thus, the archive may represent an important resource for bringing together researchers and patients who can foster PCOR principles (e.g., reciprocal relationships, co-learning, partnership, trust, transparency, honesty, respect) [20,21] and learn from each other's perspectives.

The study's patient-engaged process may have contributed to the high satisfaction data. For example, patient input emphasized the need for convenience in narrative collection (e.g., by including telephone interviews, not requiring participants to participate with a friend or family member and providing a mechanism for participants to approve recordings). Patient/caregiver stakeholder input also added design elements such as the value of including caregiver perspectives and the importance of being able to post stories to the web. Sharing stories between patients was felt to be important so that others could learn from the storytellers' experiences and/or not feel alone in navigating health problems. Furthermore, the medical literature suggests that patients may benefit from story sharing $[13,14,22,23]$. For example, narrative resources may help reduce the stigma associated with illness [22] and storytelling may help patients to reestablish a sense of identity, which can be disrupted by illness [14,22]. Likewise, storytelling has been found to alleviate distress among surrogates who have shared a patient's intensive care unit experience [23].

While the sample is diverse in terms of age and sex the narrative collection is limited by the under-representation of patients and caregivers with lower education levels and minority racial/ethnic backgrounds. The sample's racial/ethnic distribution is similar to Allegheny County, where the audio booth has been housed. To expand the sample's racial/ethnic and geographic diversity and the archive's overall size, our team plans to extend story collection to additional sites, including story collection in coordination with other PCORnet networks and community-based patient organizations, as well as at other PaTH Clinical Data Research Network sites. Improved outreach to under-represented minority groups (e.g., African American, Latino) with storytelling traditions is particularly important as these cultural groups may find the use of narratives to be a compelling approach to communication [24]. This hypothesis is supported by narrative interventions that have promoted behavior change in African American and Latino samples $[11,13,25]$. Furthermore, narratives have been found to be helpful for conveying information among low-literacy populations [26,27].

Users of the narrative archive should recognize that stories may not be literal recountings of events. Narrators make choices about the stories they tell and these choices are shaped by societal influences as well as personal goals [28]. In some ways, narratives may resemble literature rather than precise records of fact (e.g., a critical minute may be described at length and a year reduced to a single sentence) [14]. The optional conversation prompts may also influence participants' choices regarding story 
content. It is also worth noting that although the MyPaTH Story Booth participants overall showed high satisfaction levels, $5 \%$ reported that the narrative-recording experience was difficult. It is possible that such reports may reflect discomfort with the discussion of distressing topics, or anxiety about determining the scope of story to share. Such possibilities emphasize the importance of adequate training for staff involved in story collection, as well as established procedures for helping participants access counseling services if desired.

\section{Conclusion}

In summary, this analysis indicates that an indexed archive of patient and caregiver stories regarding health and accessing the healthcare system reflects diverse topics that are of relevance to PCOR researchers. It represents a unique resource for fostering the development of patientcentered research. We expect that the value of the archive will grow as its breadth expands, since specific topics of interest are not only more likely to be addressed - but to also be represented by multiple perspectives. In addition, the majority of storytelling participants have indicated an interest in learning of research or stakeholder engagement opportunities. As a result, the MyPaTH Story Booth archive can support the development of research teams that engage relevant stakeholders as partners throughout the research process. Such patient-centered research may hold promise for improving the quality of health and healthcare delivery.

\section{Acknowledgements and Conflicts of Interest}

This article was funded through a Patient-Centered Outcomes Research Institute (PCORI) Award (PCORI CDRN \#1306-04912) for development of the National Patient-Centered Clinical Research Network, known as PCORnet. The views, statements and opinions presented in this article are solely the responsibility of the authors and do not necessarily represent the views of the PatientCentered Outcomes Research Institute (PCORI), its Board of Governors or Methodology Committee or other participants in PCORnet. The sponsor had no role in the study design beyond specifying that the funding was intended to engage health system leaders to prioritize research topics. PCORI had no role in the collection, analysis or interpretation of data, writing of the report, or decision to submit the manuscript for publication. The authors declare no conflicts of interest.

\section{References}

[1] Slutsky, J., Sheridan, S. \& Selby, J. (2014). "Getting engaged". Journal of General Internal Medicine 29 (12) 1582-1583.
[2] Pignone, M. (2012). Challenges to implementing patient-centered research. Annals of Internal Medicine 157 (6) $450-451$.

[3] Forsythe, L.P., Ellis, L.E., Edmundson, L., Sabharwal, R., Rein, A., Konopka, K. \& Frank, L. (2016). Patient and Stakeholder Engagement in the PCORI Pilot Projects: Description and Lessons Learned. Journal of General Internal Medicine 31 (1) 13-21.

[4] Callon, M., Lascoumes, P. \& Barthe, Y. (2009). Acting in an Unertain World: An Essay on Technical Democracy. Cambridge, MA: MIT Press.

[5] Workman, T., Maurer, M. \& Carman, K. (2013). Unresolved tensions in consumer engagement in CER: a US research perspective. Journal of Comparative Effectiveness Research 2 (2) 127-134.

[6] Charon, R. (2001). Narrative medicine: form, function, and ethics. Annals of Internal Medicine 134 (1) 83-87.

[7] Greenhalgh, T. \& Hurwitz, B. (1999). Narrative based medicine: why study narrative? British Medical Journal 318 (7175) 48-50.

[8] Rose, R., Chakraborty, S., Mason-Lai, P., Brocke, W., Page, S.A. \& Cawthorpe, D. (2016). The storied mind: A meta-narrative review exploring the capacity of stories to foster humanism in health care. Journal of Hospital Administration 1, 52-61.

[9] Nass, P., Levine, S. \& Yancy, C. (2012). Methods for involving patients in topic generation for patient-centered comparative effectiveness research: An international perspective. Research Priorities White Paper (PCORISOL-RPWG-001) for the Patient-Centered Outcomes Research Institute (PCORI). Washington (DC): PCORI.

[10] Evans, M., Shaw, A. \& Sharp, D. (2012). Integrity in patients' stories: 'Meaning-making' through narrative in supportive cancer care. European Journal of Integrative Medicine 4, e11-e18.

[11] Houston, T.K., Allison, J.J., Sussman, M., Horn, W., Holt, C.L., Trobaugh, J., Salas, M., Pisu, M., Cuffee, Y.L., Larkin, D., Person, S.D., Barton, B., Kiefe, C.I. \& Hullett, S. (2011). Culturally appropriate storytelling to improve blood pressure: a randomized trial. Annals of Internal Medicine 154 (2) 77-84.

[12] Campbell, T., Dunt, D., Fitzgerald, J.L. \& Gordon, I. (2015). The impact of patient narratives on self-efficacy and self-care in Australians with type 2 diabetes: stage 1 results of a randomized trial. Health Promotion International 30 (3) 438-448.

[13] Goddu, A.P., Raffel, K.E. \& Peek, M.E. (2015). A story of change: The influence of narrative on AfricanAmericans with diabetes. Patient Education and Counseling 98 (8) 1017-1024.

[14] Begley, A., Pritchard-Jones, K., Biriotti, M., Kydd, A., Burdsey, T. \& Townsley, E. (2014). Listening to patients with cancer: using a literary-based research method to understand patient-focused care. BMJ Open 4 (10) e005550.

[15] Janssen, A.L. \& MacLeod, R.D. (2010). What can people approaching death teach us about how to care? Patient Education and Counseling 81 (2) 251-256.

[16] Kumagai, A.K., Murphy, E.A. \& Ross, P.T. (2009). Diabetes stories: use of patient narratives of diabetes to 
teach patient-centered care. Advances in Health Sciences Education Theory and Practice 14 (3) 315-326.

[17] Swift, T.L. \& Dieppe, P.A. (2005). Using expert patients' narratives as an educational resource. Patient Education and Counseling 57 (1) 115-121.

[18] Amin, W., Tsui, F.R., Borromeo, C., Chuang, C.H., Espino, J.U., Ford, D. et al. (2014). PaTH: towards a learning system in the Mid-Atlantic region. Journal of the American Medical Informatics Association 21 (4) 633-636. [19] US National Library of Medicine. Health Topics 2017. Available at:

https://medlineplus.gov/healthtopics.html.

[20] Frank, L., Forsythe, L., Ellis, L., Schrandt, S., Sheridan, S., Gerson, J., Konopka, K. \& Daugherty, S. (2015). Conceptual and practical foundations of patient engagement in research at the patient-centered outcomes research institute. Quality of Life Research 24 (5) 10331041.

[21] Mackie, T.I., Sheldrick, R.C., de Ferranti, S.D., Saunders, T., Rojas, E.G. \& Leslie, L.K. (2017). Stakeholders' Perspectives on Stakeholder-engaged Research (SER): Strategies to Operationalize Patientcentered Outcomes Research Principles for SER. Medical Care 55 (1) 19-30.

[22] Atkinson, S. \& Rubinelli, S. (2012). Narrative in cancer research and policy: voice, knowledge and context. Critical Reviews in Oncology/Hematology 84 (Supplement 2) S11-S16.

[23] Schenker, Y., Dew, M.A., Reynolds, C.F., Arnold, R.M., Tiver, G.A. \& Barnato, A.E. (2015). Development of a post-intensive care unit storytelling intervention for surrogates involved in decisions to limit life-sustaining treatment. Palliative \& Supportive Care 13 (3) 451-463.

[24] Palacios, J.F., Salem, B., Hodge, F.S., Albarran, C.R., Anaebere, A. \& Hayes-Bautista, T.M. (2015). Storytelling: A Qualitative Tool to Promote Health Among Vulnerable Populations. Journal of Transcultural Nursing 26 (4) 346353.

[25] Larkey, L.K., Lopez, A.M., Minnal, A. \& Gonzalez, J. (2009). Storytelling for promoting colorectal cancer screening among underserved Latina women: a randomized pilot study. Cancer Control 16 (1) 79-87.

[26] Moran, M.B., Frank, L.B., Chatterjee, J.S., Murphy, S.T. \& Baezconde-Garbanati, L. (2016). A pilot test of the acceptability and efficacy of narrative and non-narrative health education materials in a low health literacy population. Journal of Communication in Healthcare 9 (1) 40-48.

[27] Michielutte, R., Bahnson, J., Dignan, M.B. \& Schroeder, E.M. (1992). The use of illustrations and narrative text style to improve readability of a health education brochure. Journal of Cancer Education 7 (3) 251-260.

[28] Shapiro, J. (2011). Illness narratives: reliability, authenticity and the empathic witness. Medical Humanities 37 (2) 68-72. 